\title{
Hydrogel-Assisted 3D Volumetric Hotspot for Sensitive Detection by Surface-Enhanced Raman Spectroscopy
}

\author{
Soo Hyun Lee ${ }^{1} \mathbb{D}$, Sunho Kim ${ }^{2}$, Jun-Yeong Yang ${ }^{1} \mathbb{D}$, ChaeWon Mun ${ }^{1}$, Seunghun Lee ${ }^{1} \mathbb{D}$, Shin-Hyun Kim ${ }^{2, * \mathbb{D}}$ \\ and Sung-Gyu Park 1,*(D)
}

1 Department of Nano-Bio Convergence, Korea Institute of Materials Science, 797 Changwondae-ro, Changwon 51508, Korea; sh_lee@kims.re.kr (S.H.L.); yjy8184@kims.re.kr (J.-Y.Y.); apple1025@kims.re.kr (C.M.); seunghun@kims.re.kr (S.L.)

2 Department of Chemical and Biomolecular Engineering, Korea Advanced Institute of Science and Technology (KAIST), Daejeon 34141, Korea; shkim1020@kaist.ac.kr

* Correspondence: kim.sh@kaist.ac.kr (S.-H.K.); sgpark@kims.re.kr (S.-G.P.)

Citation: Lee, S.H.; Kim, S.; Yang J.-Y.; Mun, C.; Lee, S.; Kim, S.-H.; Park, S.-G. Hydrogel-Assisted 3D Volumetric Hotspot for Sensitive Detection by Surface-Enhanced Raman Spectroscopy. Int. J. Mol. Sci. 2022, 23, 1004. https://doi.org/ $10.3390 /$ ijms 23021004

Academic Editor: David Mills

Received: 2 December 2021

Accepted: 11 January 2022

Published: 17 January 2022

Publisher's Note: MDPI stays neutral with regard to jurisdictional claims in published maps and institutional affiliations.

Copyright: (C) 2022 by the authors. Licensee MDPI, Basel, Switzerland. This article is an open access article distributed under the terms and conditions of the Creative Commons Attribution (CC BY) license (https:// creativecommons.org/licenses/by/ $4.0 /)$.

\begin{abstract}
Effective hotspot engineering with facile and cost-effective fabrication procedures is critical for the practical application of surface-enhanced Raman spectroscopy (SERS). We propose a SERS substrate composed of a metal film over polyimide nanopillars (MFPNs) with three-dimensional (3D) volumetric hotspots for this purpose. The 3D MFPNs were fabricated through a two-step process of maskless plasma etching and hydrogel encapsulation. The probe molecules dispersed in solution were highly concentrated in the 3D hydrogel networks, which provided a further enhancement of the SERS signals. SERS performance parameters such as the SERS enhancement factor, limit-of-detection, and signal reproducibility were investigated with Cyanine5 (Cy5) acid Raman dye solutions and were compared with those of hydrogel-free MFPNs with two-dimensional hotspots. The hydrogel-coated MFPNs enabled the reliable detection of Cy5 acid, even when the Cy5 concentration was as low as $100 \mathrm{pM}$. We believe that the 3D volumetric hotspots created by introducing a hydrogel layer onto plasmonic nanostructures demonstrate excellent potential for the sensitive and reproducible detection of toxic and hazardous molecules.
\end{abstract}

Keywords: surface-enhanced Raman spectroscopy; volumetric hotspot engineering; maskless plasma etching; hydrogel encapsulation

\section{Introduction}

Surface-enhanced Raman spectroscopy (SERS) is a powerful and promising technique for identifying molecular fingerprints corresponding to molecules' vibrational energy states, enabling rapid, contactless, sensitive, label-free, and reliable chemical and biomedical analyses [1-7]. The amplification of Raman signals originates predominantly from an interaction of incident light with excited electron clouds of noble-metal (e.g., Ag, $\mathrm{Au}$, and $\mathrm{Cu}$ ) nanostructures (NSs), which is known as the localized surface plasmon resonance (LSPR) effect. Therefore, extensive effort has been devoted to designing elaborate plasmonic hotspots with sub-10 nm scales [8-13]. Despite its outstanding sensitivity, the SERS technique has not been widely adopted in practical fields because of complicated fabrication processes, high costs, and poor signal uniformity and reproducibility.

Maskless plasma etching has emerged as an alternative to existing high-precision and complex nanopatterning methods because of its simplicity, cost-effectiveness, lowtemperature process, and excellent throughput [14-17]. For polymer substrates in particular, this technique is favorable for fabricating NSs with high-areal density such as nanotunnels [18], nanodimples [19,20], and nanopillars [17,21] without mask patterning. The formation of such NSs is fundamentally related to complex surface dynamics, including crystallinity-dependent etching, surface migration, agglomeration, and coalescence [22,23]. The geometric properties of NSs are also conveniently tunable through adjustment of the 
radio-frequency $(\mathrm{RF})$ power, operating pressure, injection gas, and processing time [21,22]. However, the structures of many commercial polymers undergo thermal deformation during SERS measurements, which limits reliable SERS analyses $[24,25]$. To overcome this problem, researchers have investigated polyimide (PI) as a polymer-based SERS substrate because of its good thermal properties (e.g., glass transition temperature of $300{ }^{\circ} \mathrm{C}$ ) and chemical/abrasion resistance [26].

Although numerous SERS platforms have been reported, the signals obtained using these platforms are generally collected from molecules adsorbed on two-dimensional (2D) surfaces in hotspots. Positioning molecules in hotspot volume gives a promise towards high sensing performance, but there has been a lack of advanced methodology creating elaborated structures with porosity and sub-nanoscale in narrow active regions. Meanwhile, hydrogels have demonstrated strong potential in molecular capturing and filtering systems because of their large void fraction, controllable mesh size and charge, and hydrophilicity [27-30]. The dynamic crosslinking under exposure is benefitable for the formation of hydrogel matrix in tiny NSs. Moreover, the high water retention capacity allows the effective transportation and adsorption of molecules dispersed in a solvent, especially water, which is highly compatible with the chemical and biomedical sensing applications. Inspired by the aforementioned factors, the desirable SERS substrates combined with hydrogel networks can provide molecular-concentrating sites for analytes in a three-dimensional (3D) volume among plasmonic NSs; in particular, they provide greater opportunity for molecular adsorption at 3D volumetric hotspots along with NSs with a high aspect ratio (e.g., nanopillars) [13,31]. Accordingly, the sensitivity and limit-of-detection (LOD) are also able to be substantially improved. The infusion of target analytes into complex mixtures through a pore-size-modified hydrogel layer also contributes to selective and multiplex sensing [32-34].

In the present work, we prepared a highly sensitive and reproducible 3D volumetric SERS platform with a hydrogel-coated metal film (Au/Ag) over PI nanopillars (MFPNs). The areal density and aspect ratio of the PI nanopillars (PNs) were optimized via a two-step plasma treatment $\left(\mathrm{CF}_{4}\right.$ plasma followed by Ar plasma). The plasmonic hotspots were formed by deposition of a metal layer of appropriate thickness. Subsequent hydrogel encapsulation enables target molecules captured in 3D hydrogel mesh as well as 2D metal surfaces, improving SERS sensitivity. The enhancement factor (EF), LOD, and reproducibility of the hydrogel-coated MFPNs were estimated using Cyanine5 (Cy5) acid dyes and compared with those of hydrogel-free MFPNs. To theoretically verify the optical characteristics of hydrogel-coated MFPNs, the electric (E)-field profile was calculated on the basis of the finite-difference time-domain (FDTD) method.

\section{Results}

\subsection{Structural and Morphological Properties of Hydrogel-Coated MFPNs}

The hydrogel-encapsulated MFPNs with the 3D volumetric hotspots were fabricated via two-step maskless plasma etching and hydrogel coating (Scheme 1). To design the highly ordered PNs using the maskless plasma etching, we preliminarily investigated the influence of etching gas on the surface morphology and wettability of a PI film (Figure S1a-c). Plasma etching is a combination of physical and chemical etching components (Figure S1d). Under $\mathrm{CF}_{4}$ plasma etching, $\mathrm{CF}_{4}$ dissociates into the fluorine $(\mathrm{F})$ atoms and $\mathrm{CF}_{x}$ radicals $(1 \leq x \leq 3)$. The $\mathrm{F}$ atoms contribute to the breaking of $\mathrm{C}-\mathrm{C} / \mathrm{C}-\mathrm{H}$ bonds, the desorption of chemically reacted areas, and the displacement of $\mathrm{C}-\mathrm{H}$ bonds to $\mathrm{C}-\mathrm{F}$ bonds, whereas the $\mathrm{CF}_{x}$ radicals participate in the deposition of a fluorinated carbon layer $[22,35,36]$. Compared with the etching rate in the lateral direction, that in the vertical direction is relatively low because of competition between the desorption and deposition processes. In addition, nonpolar $\mathrm{C}-\mathrm{F}_{x}$ bonds tend to inhibit interaction with water molecules. Under Ar plasma etching, Ar atoms/ions collide with the surface atoms and then break their structures, a process referred to as sputtering. The potential-driven Ar atoms/ions (i.e., directionality) attribute to the formation of anisotropic NSs. The polymer atoms with broken bonds at exposed 
areas represent polarity, resulting in hydrophilicity. Such a phenomenon is consistent with experimental results. The areal density and contact angle of the $\mathrm{CF}_{4}$-plasma-etched PNs were approximately $135 \pm 10 \mu^{-2}$ and $54.1^{\circ}$, whereas those of the Ar-plasma-etched PNs were $271 \pm 16 \mu \mathrm{m}^{-2}$ and $4.3^{\circ}$, respectively. With the two-step maskless plasma etching $\left(\mathrm{CF}_{4}\right.$ plasma and subsequent Ar plasma), PNs with substantial areal density $\left(30 \pm 2 \mu \mathrm{m}^{-2}\right)$, a hydrophilic surface $\left(4.1^{\circ}\right)$, and a high aspect ratio were obtained and subsequently used as SERS substrates.

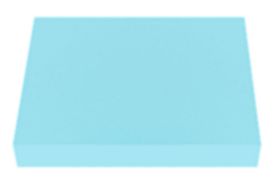

PI film

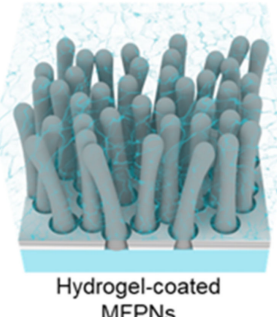

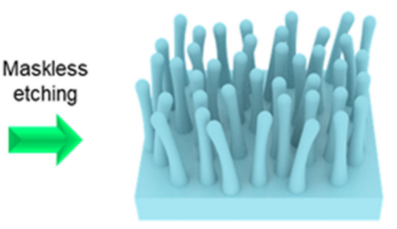

PNs
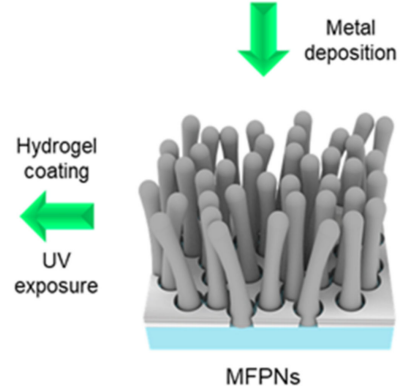

Scheme 1. Fabrication procedure for the hydrogel-coated MFPN SERS platform.

Figure 1a,b shows field-emission scanning electron microscopy (FE-SEM) images of the MFPN SERS platform without and with the hydrogel skin. The optimal PNs were obtained over a large area (Figure 1a). Their areal density and aspect ratio were predominantly controlled by the $\mathrm{CF}_{4}$ plasma and Ar plasma, respectively. After the metal deposition and hydrogel encapsulation, NSs were retained without substantial deformation or degradation (Figure 1b), which we attributed to the excellent structural adaption of the Ag and hydrogel networks. The crystalline and compositional properties of MFPNs were confirmed by field-effect transmission electron microscopy (FE-TEM) observations (Figure 1c,d). Approximately $1 \mu \mathrm{m}$-thick hydrogel completely covered the MFPNs and infiltrated the 3D interstitial space between the MFPNs. We also observed that the thermally deposited Ag layer covered the surface of the PNs conformally and smoothly. The formation of metal NSs is closely related to cohesive energy. In general, the matter is becoming softer when its cohesive energy or surface energy decreases. The cohesive energy and surface energy of $\mathrm{Ag}$ were $\sim 2.95 \mathrm{eV}$ and $\sim 1.20 \mathrm{~J} \mathrm{~m}^{-2}$, respectively, whereas those of Au were $3.81 \mathrm{eV}$ and $1.54 \mathrm{~J} \mathrm{~m}^{-2}$, respectively, indicating that the $\mathrm{Ag}$ has the better structural adaptive potential $[37,38]$. The PNs with high surface energy (hydrophilic) were wetted by the Ag layer as soft matter. The high crystallinity of the Ag layer was confirmed by the regular lattice fringes with a $d$-spacing of $2.35 \AA$, corresponding to the (111) plane of Ag (Figure 1e). The bright dot patterns also indicated the single-crystalline nature (Figure 1f). No indication of Au clusters was observed because (i) the actual Au thickness of $\leq 2 \mathrm{~nm}$ was insufficient for the formation of crystalline structures and (ii) the Au seeds were immersed by the diffused Ag layer. These characteristics were further confirmed by the elemental mapping results (Figure $1 \mathrm{~g}$ ). Both $\mathrm{C}$ and $\mathrm{O}$ were observed in the hydrogel and the PI. The plasmonic resonance of the MFPNs was confirmed by dark-field optical microscopy. LSPR excitation of plasmonic NSs is known to induce more efficient resonant Rayleigh scattering [39-41]. Vivid and different colors were observed, corresponding to wavelength-selective Rayleigh scattering under light illumination. Upon excitation of the plasmonic resonance, strong Rayleigh scattering around MFPNs was generated and could be detected in the far-field (Figure 1h). Because of structural heterogeneities of the MFPNs, broad Rayleigh scattering 
spectra were recorded across the entire visible wavelength range, whereas no scattering effect was observed over the PN substrate. The induced LSPR effect was also theoretically investigated based on the FDTD method. The geometrical parameters of simulation model were extracted from the scanning TEM (STEM) image (Figure 1g). It was found that the height of MFPNs and Ag layer (bottom) was estimated to be $350 \mathrm{~nm}$ and $150 \mathrm{~nm}$, respectively. The MFPNs were inclined with respect to the substrate surface and their interstitial distance (i.e., hotspot) was about $\leq 5 \mathrm{~nm}$. The $150 \mathrm{~nm}$ thick Ag layer did not allow the transmission and confinement of incident light due to the shallow skin depth $(2.9 \mathrm{~nm}$ at $633 \mathrm{~nm}$ ). The hotspot was realized by the PNs covered by the Ag with structural randomness. The designed model with simplified parameters demonstrated good agreement with the STEM image. As a result, a strongly confined $E$-field in 3D volumetric hotspots between the MFPNs was observed (Figure 1i). Notably, the interstitial nanogaps among high-aspect-ratio plasmonic nanopillars created a strong $E$-field concentration along the $z$-direction, indicating that the maximum $\left|E_{\mathrm{loc}}\right| /\left|E_{0}\right|$ is 52.2. Herein, the $\left|E_{\mathrm{loc}}\right|$ is the confined $E$-field intensity in hotspot and $\left|E_{0}\right|$ is the incident wave intensity. Therefore, the molecular concentration at the hotspot regions is critical to enhancing the SERS signal and the corresponding sensitivity of the SERS sensors.
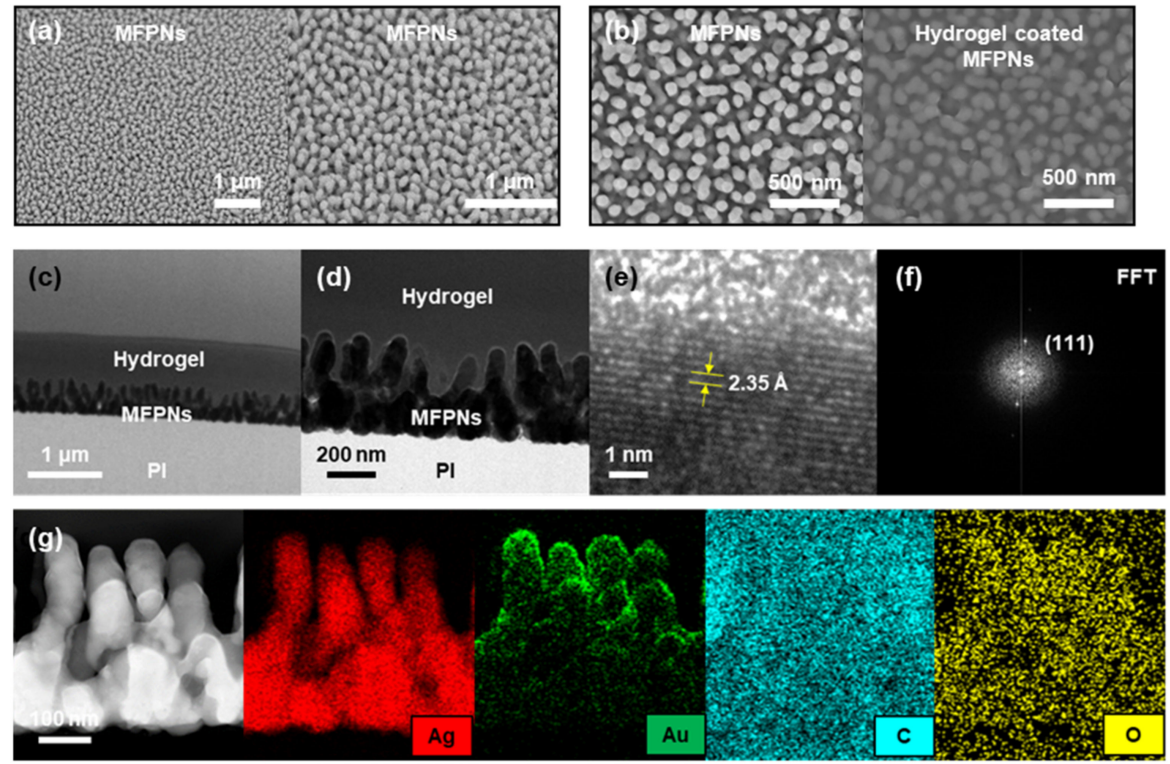

(h)

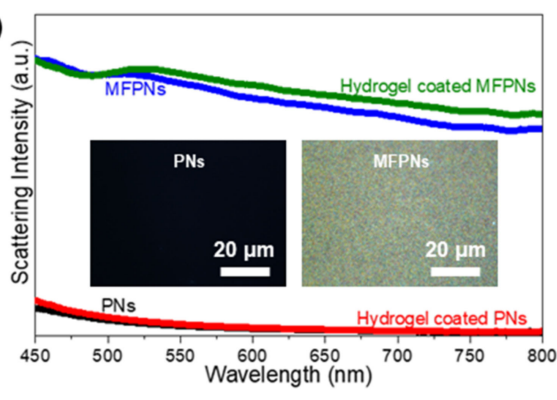

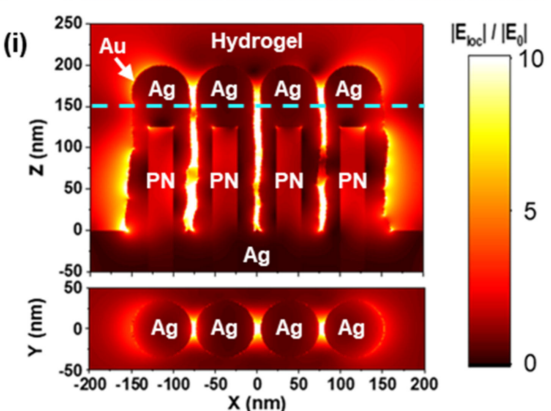

Figure 1. Morphological and crystalline analyses of the hydrogel coated metal film (Au/Ag) over polyimide (PI) nanopillars (PNs)(MFPNs). (a,b) FE-SEM images of the MFPNs without and with hydrogel encapsulation. Structural and crystalline properties of the hydrogel-coated MFPNs observed by (c,d) FE-TEM images, (e) HR-TEM image, and (f) the fast Fourier transform of the image in (e). (g) Compositional analysis with elemental mapping of $\mathrm{Ag}, \mathrm{Au}, \mathrm{C}$, and $\mathrm{O}$ in the hydrogel-coated MFPNs. (h) Scattering intensity of the nonplasmonic PNs and plasmonic MFPNs with and without the hydrogel skin. The photograph of (h) demonstrates the true color of scattered light. (i) Numerically calculated E-field profiles of the hydrogel-coated MFPNs. 


\subsection{SERS Activities of Hydrogel-Coated MFPNs}

We investigated the SERS performance of the hydrogel-coated MFPNs by analyzing the quantitative Raman signals of the Cy5 acid (Figure 2a,b). The dye solutions were prepared over six decades of dilution $(10 \mathrm{pM}$ to $10 \mu \mathrm{M})$. The spectral intensity was gradually decreased with decreasing dye concentration. The characteristic spectral features of the Cy5 acid were clearly detected even at the low concentration of $100 \mathrm{pM}$. Among these features, the most intense peak at $1352 \mathrm{~cm}^{-1}$, corresponding to typical methine chain vibrations of indocarbocyanines [42,43], was attributed to a representative peak and was used for further analyses. The SERS EF of hydrogel-coated MFPNs was evaluated on the basis of the Raman intensity ratio between the plasmonic and nonplasmonic structures (Figure 2c). The EF was calculated by the following equation [7]:

$$
\mathrm{EF}=\left(\frac{I_{\text {SERS }}}{I_{\text {bare }}}\right)\left(\frac{N_{\text {bare }}}{N_{\text {SERS }}}\right)
$$

Umber of molecules producing the $I_{\text {SERS }}$ and $I_{\text {bare }}$ intensities, respectively. Under the assumption of homogeneous distributions of $\mathrm{Cy} 5$ acid dyes over the surfaces, the average number of molecules $(N)$ can be described using the equation of

$$
N=\left(N_{\mathrm{A}} \times c \times \frac{V}{A_{\text {sub }}}\right) A_{\text {laser }}
$$

where $N_{\mathrm{A}}, c, V, A_{\mathrm{sub}}$ and $A_{\text {laser }}$ are Avogadro's constant, the dye concentration, the volume of Cy5 acid solution, the surface area covered by the solution, and the area of the laser spot, respectively. Herein, the same experimental conditions were used for both the SERS and bare platforms; thus, the parameters for $N_{\mathrm{A}}, V, A_{\mathrm{sub}}$, and $A_{\text {laser }}$ could be discarded. The equation for $\mathrm{EF}$ could therefore be simplified as

$$
\mathrm{EF}=\left(\frac{I_{\text {SERS }}}{I_{\text {bare }}}\right)\left(\frac{c_{\text {bare }}}{c_{\text {SERS }}}\right) .
$$

For the bare signal, the Raman spectrum for hydrogel-coated PNs dipped in $100 \mu \mathrm{M}$ Cy5 acid solution was recorded. At $1352 \mathrm{~cm}^{-1}, I_{\text {SERS }}$ and $I_{\text {bare }}$ were estimated to be 52 and 25, respectively, resulting in an experimental EF value of $2.1 \times 10^{6}$ (Figure 2c). Because the EF is approximately proportional to the fourth power of the $E$-field, on the basis of Figure 1i, the theoretical EF was evaluated to be $7.4 \times 10^{6}$. Reasonable EFs were obtained despite the difficulty in matching the geometric and optical parameters between the actual and modeled structures. To validate the hydrogel functionality, we compared these behaviors with those of hydrogel-free MFPNs (Figure 2d). A comparable tendency related to the concentration of the Cy5 acid was observed. The hydrogel-coated MFPNs exhibited excellent sensitivity (EF of $2.1 \times 10^{6}$ and LOD of $100 \mathrm{pM}$ ) compared with the hydrogel-free MFPNs (EF of $3.7 \times 10^{4}$ and LOD of $10 \mathrm{nM}$ ). 

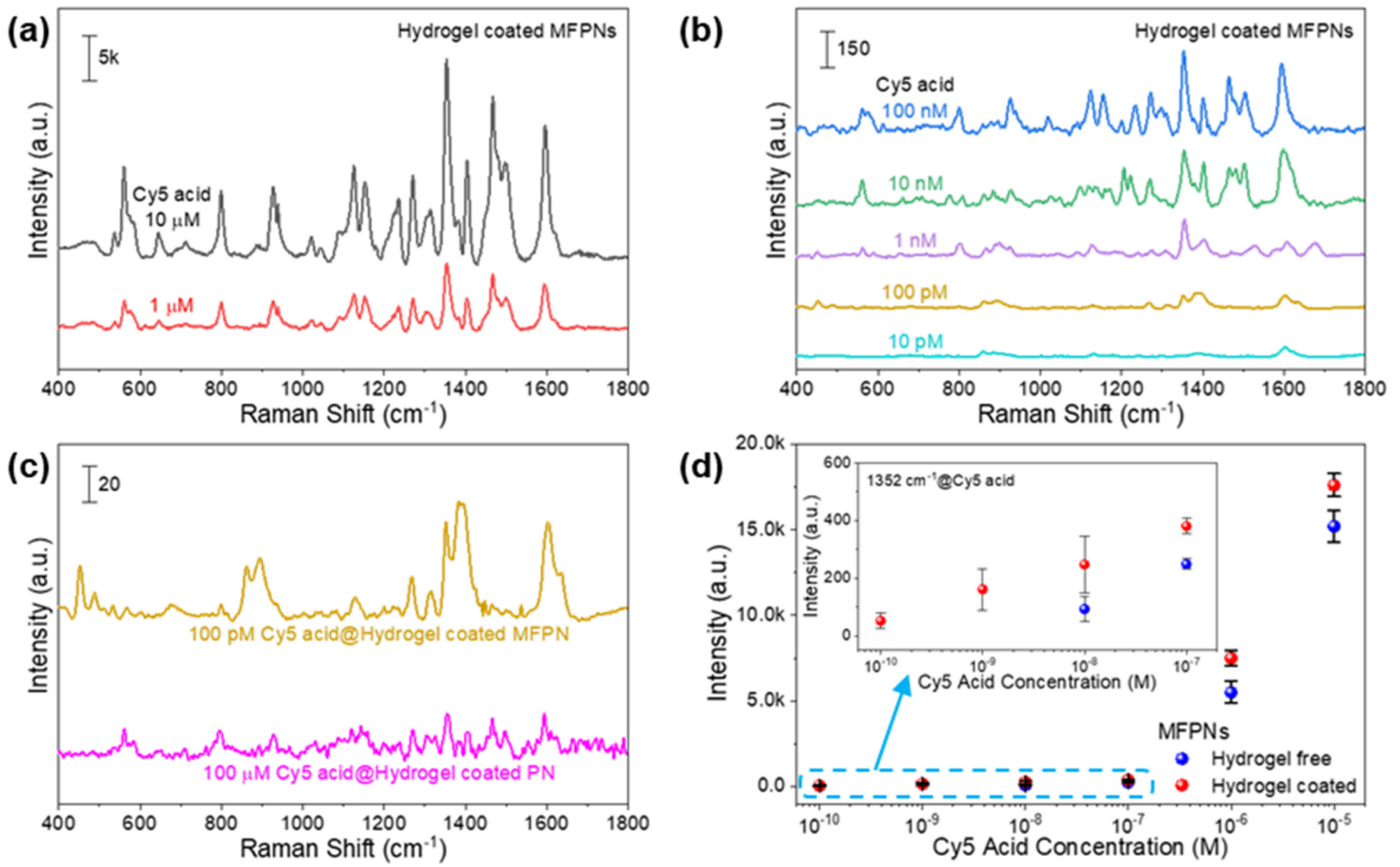

Figure 2. Sensitivity and LOD of the metal film (Au/Ag) over polyimide nanopillars (PNs)(MFPNs) with and without the hydrogel skin. Quantitative SERS analysis of the hydrogel-coated MFPNs for Cyanine5 (Cy5) acid concentrations of (a) 1-10 $\mu \mathrm{M}$ and (b) $10 \mathrm{pM}$ to $100 \mathrm{nM}$. (c) Raman spectra of the plasmonic and nonplasmonic substrates encapsulated with hydrogel matrix. (d) Quantitative Raman intensity at $1352 \mathrm{~cm}^{-1}$ between the MFPNs with and without the hydrogel layer in Cy5 acid at concentrations ranging from $100 \mathrm{pM}$ to $10 \mu \mathrm{M}$.

\subsection{Reproducibility of Hydrogel-Coated MFPNs}

Practical assays require homogeneous SERS signals that arise from any random spot on a plasmonic structure. The spectra for Cy5 acid on MFPNs with and without the hydrogel skins were acquired using a Raman mapping image system equipped with an $x-y$ stage remotely controlled by a computer (Figure 3a). The mapping scan was performed in an area of $25 \times 25 \mu \mathrm{m}^{2}$ in intervals of $2.5 \mu \mathrm{m}$ (the interval should be larger than laser spot diameter of $1.9 \mu \mathrm{m})$. For the $10 \mu \mathrm{M}$ Cy 5 acid on hydrogel-coated MFPNs, the overall spectra (100 points) displayed high uniformity through the entire measurement range (Figure 3b). At the characteristic peak of $1352 \mathrm{~cm}^{-1}$, the Raman intensities were distributed within two standard deviations from the average (Figure 3c). On the basis of these intensities, the signals with a relative standard deviation (RSD) of $10.0 \%$ exhibited the excellent reproducibility of hydrogel-coated MFPNs. This was also confirmed in the reconstructed Raman mapping image in Figure 3d. For comparison, Raman mapping was also performed for $10 \mu \mathrm{M}$ Cy5 acid on hydrogel-free MFPNs (Figure 3e). The Raman mapping for the hydrogel-free MFPNs indicated uniform signals with an RSD of 9.0\%. This strong signal stability of MFPNs was attributed to the reasonably high periodicity of the PNs prepared by maskless plasma etching. 
(a)

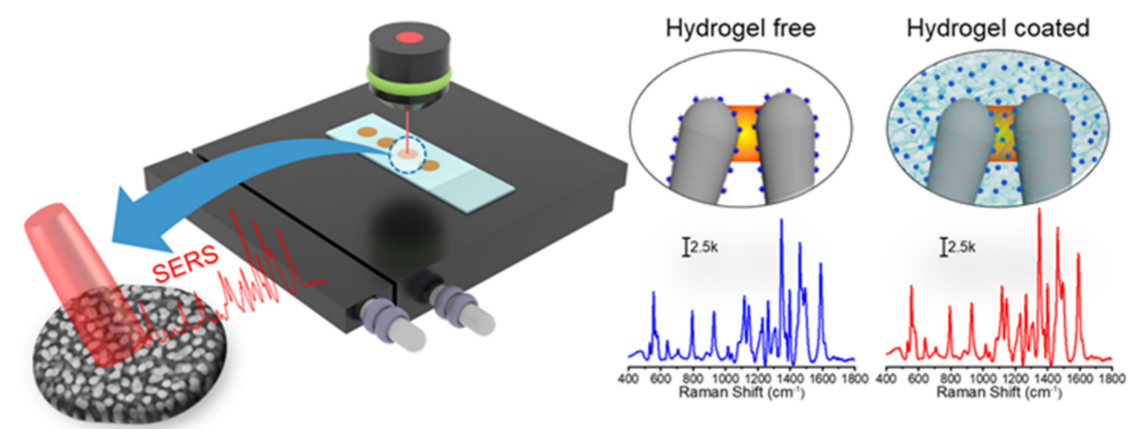

(b)
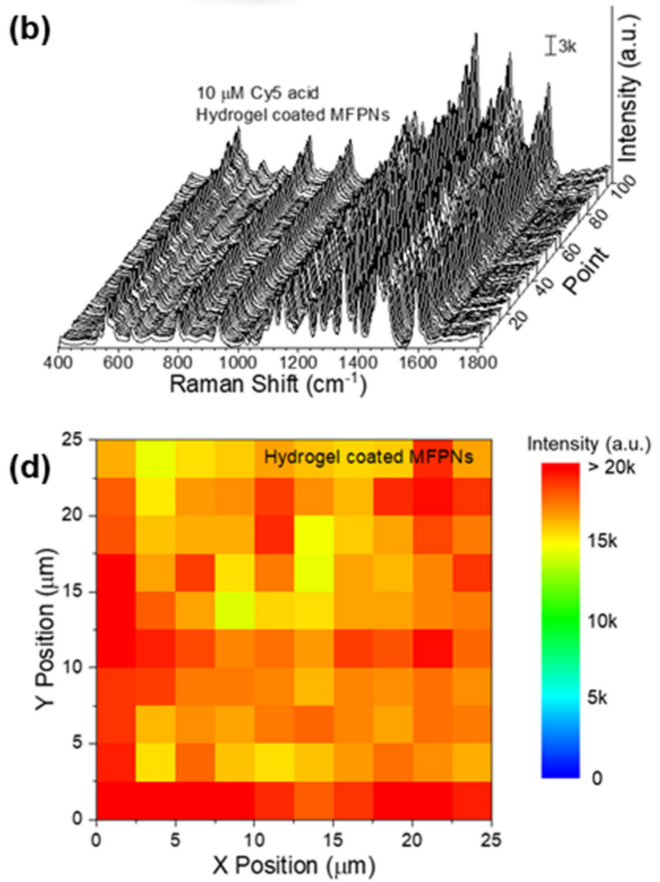

(c)

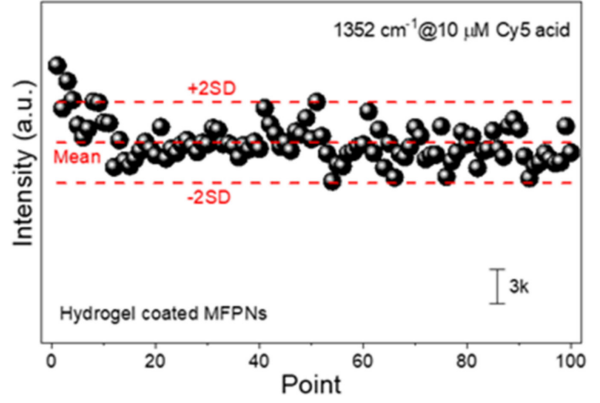

(e)

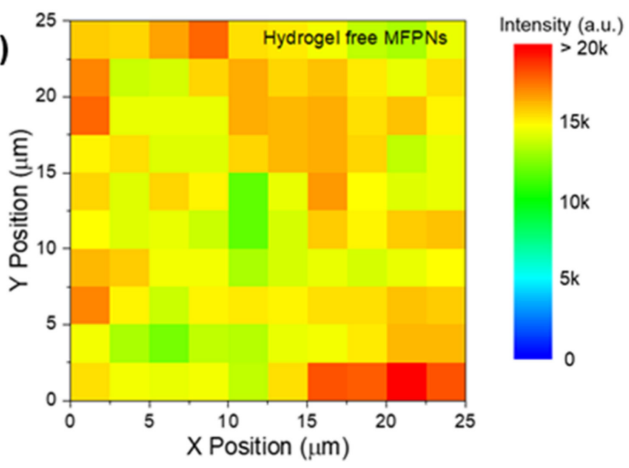

Figure 3. Reproducibility of metal film (Au/Ag) over polyimide nanopillars (MFPNs) with and without the hydrogel skin. (a) Strategic diagram of the systematical measurement for Raman mapping. (b) Raman mapping spectra and (c) corresponding Raman intensities at $1352 \mathrm{~cm}^{-1}$ of $10 \mu \mathrm{M}$ Cyanine5 (Cy5) acid on hydrogel-coated MFPNs. Contour plot for the Raman mapping spectra of $10 \mu \mathrm{M} \mathrm{Cy} 5$ acid on MFPNs (d) with and (e) without hydrogel encapsulation.

\subsection{Optimization of Dipping Time for Efficient SERS Analysis}

The SERS signals are proportional to the probe molecules adsorbed in the hotspots. For the MFPNs with and without the hydrogel layer, we optimized the dipping time to achieve high SERS performance in a relatively short time. The SERS intensities of the MFPNs with and without the hydrogel were measured at $1352 \mathrm{~cm}^{-1}$ after the MFPNs were immersed in $10 \mu \mathrm{M}$ Cy5 acid solution for various times $(t=15,30,45,60 \mathrm{~min}$ ) (Figure 4 ). For both the MFPNs with and without the hydrogel matrix, with increasing dipping time, the peak intensity increased, whereas the slope efficiency $\left(I_{\text {Raman }} / t\right)$ was gradually degraded. The intensity almost reached saturation when the dipping time was $60 \mathrm{~min}$. Therefore, the optimum dipping time was set to be $60 \mathrm{~min}$. We also observed that the Raman intensity of the hydrogel-coated MFPNs was consistently higher than that of the hydrogel-free MFPNs because the hydrophilic hydrogel networks allowed the effective diffusion of dyes into the $3 \mathrm{D}$ volumetric hotspots. 


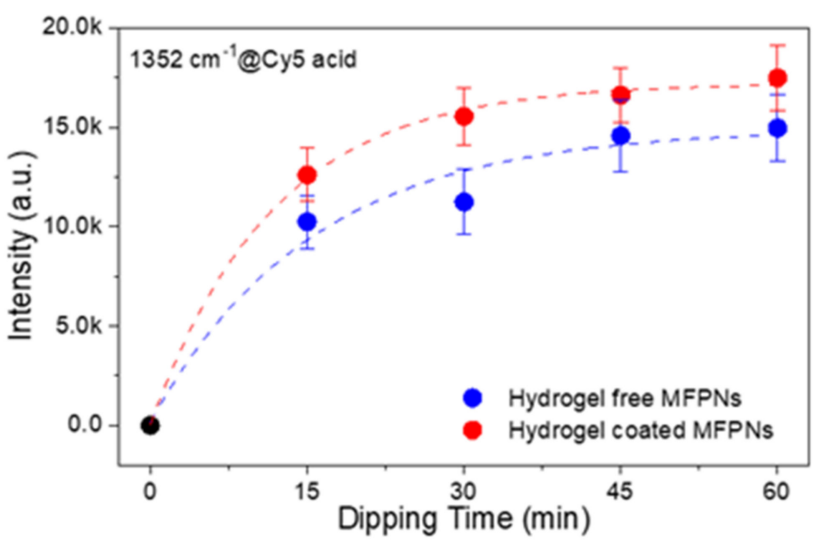

Figure 4. Variations of the Raman intensity at $1352 \mathrm{~cm}^{-1}$ of $10 \mu \mathrm{M}$ Cyanine 5 (Cy5) acid on metal film $(\mathrm{Au} / \mathrm{Ag})$ over polyimide (PI) nanopillars (MFPNs) with and without the hydrogel layer, plotted as a function of the dipping time.

\section{Discussion}

The development of hotspots with high coherency and density is a key factor governing the SERS activity. The maskless plasma etching is considered a convenient fabrication method for polymer NSs with excellent tunability in geometry (i.e., shape, areal density, and aspect ratio) and wettability. Since a hotspot is defined as the dielectric spaces between the plasmonic NSs in proximity (typically sub-10 nm), the areal density of NSs is directly associated with the hotspot density as well as the SERS sensitivity. Despite structural randomness, a remarkable hotspot population also provides an opportunity to increase the order of regularity in NS arrays $[7,13,19,44]$. Consequently, it is available to achieve SERS signals with high uniformity, leading to sensitive, reproducible, repeatable, and reliable molecular detection. In the present study, the highly populated MFPNs $\left(30 \pm 2 \mu \mathrm{m}^{-2}\right)$ obtained by our novel two-step maskless plasma treatment method demonstrated excellent SERS spectral stability, with an RSD of $9.0 \%$ (Figure 3e).

The aspect ratio of the NSs and hydrogel attributed to the formation of 3D volumetric hotspots. Although the pillar-like structures produce strongly confined E-fields along their longitudinal axis, the modified surface tension across the narrow regions inhibits effective molecular penetration. To characterize the molecular transport in addition to the volumetric distributions, we used the hydrogel matrix. In the present study, the MFPNs with a high aspect ratio (Figures $1 \mathrm{~g}$ and S1c) were conformally wetted by the Ag layer (Figure $1 \mathrm{~b}-\mathrm{d}, \mathrm{g}$ ), which acted as soft matter because of its intrinsic material properties. The plasmonic activities of MFPNs were experimentally (Figure 1h) and theoretically (Figure 1i) demonstrated. The hydrogel solution was diluted in ethanol for better infiltration, resulting in MFPNs fully covered with hydrogel networks (Figure $1 \mathrm{~b}-\mathrm{d}$ ). The solute diffusion through hydrogel is described with the following three traditional theories: hydrodynamic theory, free volume theory, and obstruction theory. The mesh size of hydrogel matrix was larger than $4.6 \mathrm{~nm}$ according to our previous study [32]. Based on the reported hydrodynamic radius of Cy5 $(0.81 \mathrm{~nm})$ [45], the diffusivity of Cy5 acid was estimated to be $269 \mu \mathrm{m}^{2} / \mathrm{s}$, which is very high enough to diffuse into the whole hydrogel thickness. Therefore, it is postulated that the Cy5 acid dyes dissolved in water were diffused into the MFPNs through the hydrogel matrix (i.e., hydrodynamic theory). This full-coverage enabled the target molecules to locate not only on the substrate surface but also in the spaces. Therefore, the introduction of a hydrogel layer into the plasmonic NSs is important for enhancing the SERS performance (i.e., the sensitivity and reproducibility). The hydrogelencapsulated MFPNs exhibited an EF of $2.1 \times 10^{6}$, LOD of $100 \mathrm{pM}$, and RSD of $10.0 \%$, as compared with those of hydrogel-free MFPNs (EF of $3.7 \times 10^{4}$, LOD of $10 \mathrm{nM}$, and RSD of 9.0\%) (Figures 2 and 3). 
In conclusion, the hydrogel-assisted MFPNs with 3D volumetric hotspots provide great insight into the development of a highly sensitive, reproducible, and reliable SERS platform for the detection and identification of small analytes.

\section{Materials and Methods}

\subsection{Fabrication Procedures of Hydrogel-Coated MFPNs}

A $50 \mu \mathrm{m}$-thick PI film with dimensions of $3 \times 3 \mathrm{~cm}^{2}$ (Isoflex Kespi, Siheung, Korea) was used as a SERS substrate after its protective film was removed. To obtain the desired PNs, a two-step maskless plasma etching technique was conducted by reactive-ion etching using 13.56 MHz RF plasma equipment (LAT, Osan, Korea). At the initial stage, the base pressure of the chamber was set to be $7.0 \mathrm{mTorr}$. The $\mathrm{CF}_{4}$ plasma treatment was applied with a gas flow rate of $3 \mathrm{sccm}$, working pressure of $50 \mathrm{mTorr}$, and RF power of $100 \mathrm{~W}$ for $120 \mathrm{~s}$. After the recovery of the base pressure, the Ar plasma etching with a gas flow rate of $3 \mathrm{sccm}$, working pressure of $30 \mathrm{mTorr}$, and RF power of $100 \mathrm{~W}$ was carried out for $120 \mathrm{~s}$. To produce the plasmonic hotspots, a metal film was deposited over the PNs, resulting in MFPNs. A $200 \mathrm{~nm}$-thick Ag layer was deposited at a rate of $1.8 \AA^{\circ} \mathrm{s}^{-1}$ using a thermal evaporator (LAT, Osan, Korea). A 5 nm-thick Au layer was subsequently deposited at a rate of $2.0 \AA \mathrm{s}^{-1}$ under an applied RF power of $100 \mathrm{~W}$ using a sputtering system (LAT, Osan, Korea). The as-fabricated MFPNs were diced into individual SERS platforms with dimensions of $5 \times 5 \mathrm{~mm}^{2}$.

A hydrogel solution was prepared by dissolving poly(ethylene glycol) diacrylate (PEGDA; MW $700 \mathrm{~g} \mathrm{~mol}^{-1}$, Sigma-Aldrich) and 1-[4-(2-hydroxyethoxy)-phenyl]-2-hydroxy2-methyl-1-propane-1-one (Irgacure 2959, BASF) as a photoinitiator in deionized (DI) water. To control the surface area of the hydrogel skin, the solution was highly diluted with ethanol. To render the hydrogel layer, a $1 \mu \mathrm{L}$ droplet $(0.7 w / w \%$ PEGDA, $0.6 w / w \%$ DI water, and $0.005 w / w \%$ photoinhibitor) was placed on the surface of MFPNs and evaporated for $5 \mathrm{~min}$ in air. Under UV exposure, the PEGDA molecules were crosslinked (i.e., via free-radical polymerization). The hydrogel-coated MFPNs were rinsed with DI water to remove the excess monomer residues and then dried in air.

\subsection{Characterization}

The morphological properties of hydrogel-coated MFPNs were observed by FE-SEM (JSM-7900F, Jeol, Tokyo, Japan). The crystallinity and elemental composition were explored via focused ion beam (Quanta 3D FEG, FEI, Eindhoven, Netherlands) milling at $0.1 \mathrm{nA}$ and $30 \mathrm{kV}$ and by FE-TEM (JEM-2100F, Jeol, Tokyo, Japan) in conjunction with energydispersive X-ray analysis (EDX) at an accelerating voltage of $200 \mathrm{kV}$. The SERS activities of the hydrogel-coated MFPNs were evaluated using a Raman microscope (HEDA, NOST, Sungnam, Korea) equipped with a $633 \mathrm{~nm}$ laser diode $(0.16 \mathrm{~mW})$ and a $20 \times$ objective lens $(\mathrm{NA}=0.45)$. For the quantitative studies, the samples were immersed into Raman probe solutions (10 pM to $10 \mu \mathrm{M})$, followed by ambient drying. Their spectra in the wavenumber range $400-1800 \mathrm{~cm}^{-1}$ were acquired for $1 \mathrm{~s}$ at five random points. $\mathrm{Cy} 5$ acid $\left(\mathrm{C}_{32} \mathrm{H}_{39} \mathrm{ClN}_{2} \mathrm{O}_{2}\right.$; $98 \%$, Broadpharm) was used as a Raman probe molecule.

\subsection{Theoretical Computation}

The $E$-field profiles across the MFPNs were theoretically calculated using the FDTD software (version 2021 R1.2, Ansys Lumerical, Seoul, Korea). The geometric parameters were extracted from the STEM image. The Ag nanopillar arrays covering PNs were depicted with surface roughness. The hydrogel skin was used as the top domain, whereas the Ag film was used as the bottom domain. For the boundary condition, the perfectly matched layer was applied to all planes to eliminate the reflection at an interface. The $E_{x}$-polarized plane wave at $\lambda=633 \mathrm{~nm}\left(E_{0}\right)$ was produced from the active port and was incident to the MFPNs in the normal direction. The intensity of $E_{0}$ was determined to be $1 \mathrm{~V} \mathrm{~m}^{-1}$. The mesh size was fixed at $0.5 \mathrm{~nm}$. For computations, the dielectric constant of the Ag and $\mathrm{Au}$ 
was taken as $\varepsilon_{\mathrm{Ag}}=-18.8626+2.3267 \mathrm{i}$ and $\varepsilon_{\mathrm{Au}}=-10.8911+0.7948$, respectively, and the refractive index of the hydrogel was set to 1.47 .

Supplementary Materials: The following supporting information can be downloaded at https: //www.mdpi.com/article/10.3390/ijms23021004/s1.

Author Contributions: Conceptualization, S.H.L. and S.-G.P.; methodology, S.H.L., S.K. and C.M.; software, J.-Y.Y.; validation, S.H.L. and S.-G.P.; investigation, S.H.L.; writing-original draft preparation, S.H.L.; writing-review and editing, S.H.L., S.L., S.-H.K. and S.-G.P.; supervision, S.-G.P.; project administration, S.-G.P.; funding acquisition, S.-G.P. All authors have read and agreed to the published version of the manuscript.

Funding: This work was supported by grants from the National Research Foundation of Korea (NRF) funded by the Ministry of Science and ICT, South Korea [Nos. 2021M3H4A1A02051036 and 2021M3H4A4079520] and the Ministry of Trade, Industry and Energy, South Korea [No. 20012405]. This research was also supported by the Korea Health Technology R\&D Project through the Korea Health Industry Development Institute (KHIDI) and Korea Dementia Research Center (KDRC), funded by the Ministry of Health \& Welfare and Ministry of Science and ICT, Republic of Korea (grant number: HU21C0335).

Institutional Review Board Statement: Not applicable.

Informed Consent Statement: Not applicable.

Data Availability Statement: Not applicable.

Conflicts of Interest: The authors declare no conflict of interest.

\section{References}

1. Laing, S.; Jamieson, L.E.; Faulds, K.; Graham, D. Surface-enhanced Raman spectroscopy for in vivo biosensing. Nat. Rev. Chem. 2017, 1, 0060. [CrossRef]

2. Hu, Y.; Cheng, H.; Zhao, X.; Wu, J.; Muhammad, F.; Lin, S.; He, J.; Zhou, L.; Zhang, C.; Deng, Y.; et al. Surface-Enhanced Raman Scattering Active Gold Nanoparticles with Enzyme-Mimicking Activities for Measuring Glucose and Lactate in Living Tissues. ACS Nano 2017, 11, 5558-5566. [CrossRef]

3. Hu, S.; Jiang, Y.; Wu, Y.; Guo, X.; Ying, Y.; Wen, Y.; Yang, H. Enzyme-Free Tandem Reaction Strategy for Surface-Enhanced Raman Scattering Detection of Glucose by Using the Composite of Au Nanoparticles and Porphyrin-Based Metal-Organic Framework. ACS Appl. Mater. Interfaces 2020, 12, 55324-55330. [CrossRef]

4. Qin, X.; Si, Y.; Wu, Z.; Zhang, K.; Li, J.; Yin, Y. Alkyne/Ruthenium(II) Complex-Based Ratiometric Surface-Enhanced Raman Scattering Nanoprobe for In Vitro and Ex Vivo Tracking of Carbon Monoxide. Anal. Chem. 2020, 92, 924-931. [CrossRef]

5. Rickard, J.J.S.; Di-Pietro, V.; Smith, D.J.; Davies, D.J.; Belli, A.; Oppenheimer, P.G. Rapid optofluidic detection of biomarkers for traumatic brain injury via surface-enhanced Raman spectroscopy. Nat. Biomed. Eng. 2020, 4, 610-623. [CrossRef]

6. Neng, J.; Zhang, Q.; Sun, P. Application of surface-enhanced Raman spectroscopy in fast detection of toxic and harmful substances in food. Biosens. Bioelectron. 2020, 167, 112480. [CrossRef]

7. Kim, W.; Lee, S.H.; Kim, J.H.; Ahn, Y.J.; Kim, Y.-H.; Yu, J.S.; Choi, S. Paper-Based Surface-Enhanced Raman Spectroscopy for Diagnosing Prenatal Diseases in Women. ACS Nano 2018, 12, 7100-7108. [CrossRef] [PubMed]

8. Lim, D.K.; Jeon, K.S.; Hwang, J.H.; Kim, H.; Kwon, S.; Suh, Y.D.; Nam, J.-M. Highly uniform and reproducible surface-enhanced Raman scattering from DNA-tailorable nanoparticles with 1-nm interior gap. Nature Nanotech. 2011, 6, 452-460. [CrossRef] [PubMed]

9. Thacker, V.V.; Herrmann, L.O.; Sigle, D.O.; Zhang, T.; Liedl, T.; Baumberg, J.J.; Keyser, U.F. DNA origami based assembly of gold nanoparticle dimers for surface-enhanced Raman scattering. Nat. Commun. 2014, 5, 3448. [CrossRef] [PubMed]

10. Montjoy, D.G.; Bahng, J.H.; Eskafi, A.; Hou, H.; Kotov, N.A. Omnidispersible Hedgehog Particles with Multilayer Coatings for Multiplexed Biosensing. J. Am. Chem. Soc. 2018, 140, 7835-7845. [CrossRef] [PubMed]

11. Zhang, Q.; Large, N.; Wang, H. Gold Nanoparticles with Tipped Surface Structures as Substrates for Single-Particle SurfaceEnhanced Raman Spectroscopy: Concave Nanocubes, Nanotrisoctahedra, and Nanostars. ACS Appl. Mater. Interfaces 2014, 6, 17255-17267. [CrossRef]

12. Tang, H.; Meng, G.; Huang, Q.; Zhang, Z.; Huang, Z.; Zhu, C. Arrays of Cone-Shaped ZnO Nanorods Decorated with Ag Nanoparticles as 3D Surface-Enhanced Raman Scattering Substrates for Rapid Detection of Trace Polychlorinated Biphenyls. Adv. Funct. Mater. 2012, 22, 218-224. [CrossRef]

13. Lin, D.; Wu, Z.; Li, S.; Zhao, W.; Ma, C.; Wang, J.; Jiang, Z.; Zhong, Z.; Zheng, Y.; Yang, X. Large-Area Au-NanoparticleFunctionalized Si Nanorod Arrays for Spatially Uniform Surface-Enhanced Raman Spectroscopy. ACS Nano 2017, 11, 1478-1487. [CrossRef] [PubMed] 
14. Xiao, D.; Ruan, Q.; Bao, D.-L.; Luo, Y.; Huang, C.; Tang, S.; Shen, J.; Cheng, C.; Chu, P.K. Effects of Ion Energy and Density on the Plasma Etching-Induced Surface Area, Edge Electrical Field, and Multivacancies in MoSe2 Nanosheets for Enhancement of the Hydrogen Evolution Reaction. Small 2020, 16, 2001470. [CrossRef] [PubMed]

15. Akinoglu, E.M.; Morfa, A.J.; Giersig, M. Understanding Anisotropic Plasma Etching of Two-Dimensional Polystyrene Opals for Advanced Materials Fabrication. Langmuir 2014, 30, 12354-12361. [CrossRef] [PubMed]

16. Leem, J.W.; Yu, J.S. Broadband and wide-angle antireflection subwavelength structures of Si by inductively coupled plasma etching using dewetted nanopatterns of Au thin films as masks. Thin Solid Films 2011, 519, 3792-3797. [CrossRef]

17. Jiang, Y.; Xu, J.; Lee, J.; Du, K.; Yang, E.-H.; Moon, M.-W.; Choi, C.-H. Nanotexturing of Conjugated Polymers via One-Step Maskless Oxygen Plasma Etching for Enhanced Tunable Wettability. Langmuir 2017, 33, 6885-6894. [CrossRef] [PubMed]

18. Her, E.K.; Chung, H.-S.; Moon, M.-W.; Oh, K.H. An angled nano-tunnel fabricated on poly(methyl methacrylate) by a focused ion beam. Nanotechnology 2009, 20, 285301. [CrossRef]

19. Dang, H.; Park, S.-G.; Wu, Y.; Choi, N.; Yang, J.-Y.; Lee, S.; Joo, S.-W.; Chen, L.; Choo, J. Reproducible and Sensitive Plasmonic Sensing Platforms Based on Au-Nanoparticle-Internalized Nanodimpled Substrates. Adv. Funct. Mater. 2021, 31, 2105703. [CrossRef]

20. Yang, J.-Y.; Park, S.-G.; Jung, S.; Byeon, E.-Y.; Kim, D.-g.; Jung, H.S.; Kim, H.J.; Lee, S. SERS substrates based on self-organized dimple nanostructures on polyethylene naphthalate films produced via oxygen ion beam sputtering. Appl. Surf. Sci. 2022, 572, 151452. [CrossRef]

21. Du, K.; Jiang, Y.; Liu, Y.; Wathuthanthri, I.; Choi, C.-H. Manipulation of the Superhydrophobicity of Plasma-Etched Polymer Nanostructures. Micromachines 2018, 9, 304. [CrossRef]

22. Phan, L.T.; Yoon, S.M.; Moon, M.-W. Plasma-Based Nanostructuring of Polymers: A Review. Polymers 2017, 9, 417. [CrossRef]

23. Ko, T.-J.; Oh, K.H.; Moon, M.-W. Plasma-Induced Hetero-Nanostructures on a Polymer with Selective Metal Co-Deposition. Adv. Mater. Interfaces 2015, 2, 1400431. [CrossRef]

24. Kho, K.W.; Shen, Z.X.; Lei, Z.; Watt, F.; Soo, K.C.; Olivo, M. Investigation into a Surface Plasmon Related Heating Effect in Surface Enhanced Raman Spectroscopy. Anal. Chem. 2007, 79, 8870-8882. [CrossRef]

25. Kang, T.; Hong, S.; Choi, Y.; Lee, L.P. The Effect of Thermal Gradients in SERS Spectroscopy. Small 2010, 6, 2649-2652. [CrossRef]

26. Gouzman, I.; Grossman, E.; Verker, R.; Atar, N.; Bolker, A.; Eliaz, N. Advances in polyimide-based materials for space applications. Adv. Mater. 2019, 31, 1807738. [CrossRef]

27. Ren, C.; Gao, Y.; Guan, Y.; Wang, Z.; Yang, L.; Gao, J.; Fan, H.; Liu, J. Carrier-Free Supramolecular Hydrogel Composed of Dual Drugs for Conquering Drug Resistance. ACS Appl. Mater. Interfaces 2019, 11, 33706-33715. [CrossRef] [PubMed]

28. Lyu, D.; Chen, S.; Guo, W. Liposome Crosslinked Polyacrylamide/DNA Hydrogel: A Smart Controlled-Release System for Small Molecular Payloads. Small 2018, 14, 1704039. [CrossRef] [PubMed]

29. Yin, H.; Yan, Z.; Bauer, R.J.; Peng, J.; Schieker, M.; Nerlich, M.; Docheva, D. Functionalized thermosensitive hydrogel combined with tendon stem/progenitor cells as injectable cell delivery carrier for tendon tissue engineering. Biomed. Mater. 2018, 13, 034107. [CrossRef] [PubMed]

30. Nele, V.; Wojciechowski, J.P.; Armstrong, J.P.K.; Stevens, M.M. Tailoring Gelation Mechanisms for Advanced Hydrogel Applications. Adv. Funct. Mater. 2020, 30, 2002759. [CrossRef]

31. Liu, J.; Ma, Y.; Shao, J.; Zhang, S.; Chen, Y. Ultra-tall sub-wavelength gold nano pillars for high sensitive LSPR sensors. Microelectron. Eng. 2018, 196, 7-12. [CrossRef]

32. Kim, S.; Choi, W.; Kim, D.J.; Jung, H.S.; Kim, D.-H.; Kim, S.-H.; Park, S.-G. Encapsulation of 3D plasmonic nanostructures with ultrathin hydrogel skin for rapid and direct detection of toxic small molecules in complex fluids. Nanoscale 2020, 12, 12942. [CrossRef]

33. Herrmann, A.; Rödiger, S.; Schmidt, C.; Schierack, P.; Schedler, U. Spatial Separation of Microbeads into Detection Levels by a Bioorthogonal Porous Hydrogel for Size-Selective Analysis and Increased Multiplexity. Anal. Chem. 2019, 91, 8484-8491. [CrossRef]

34. Sun, M.; Bai, R.; Yang, X.; Song, J.; Qin, M.; Suo, Z.; He, X. Hydrogel Interferometry for Ultrasensitive and Highly Selective Chemical Detection. Adv. Mater. 2018, 30, 1804916. [CrossRef] [PubMed]

35. Mundo, R.D.; Troia, M.; Palumbo, F.; Trotta, M.; d'Agostino, R. Nano-texturing of Transparent Polymers with Plasma Etching: Tailoring Topography for a Low Reflectivity. Plasma Process. Polym. 2012, 9, 947-954. [CrossRef]

36. Resnik, M.; Zaplotnik, R.; Mozetic, M.; Vesel, A. Comparison of $\mathrm{SF}_{6}$ and $\mathrm{CF}_{4}$ Plasma Treatment for Surface Hydrophobization of PET Polymer. Materials 2018, 11, 311. [CrossRef]

37. Wang, B.; Liu, M.; Wang, Y.; Chen, X. Structures and Energetics of Silver and Gold Nanoparticles. J. Phys. Chem. C 2011, 115, 11374-11381. [CrossRef]

38. Grouchko, M.; Roitman, P.; Zhu, X.; Popov, I.; Kamyshny, A.; Su, H.; Magdassi, S. Merging of metal nanoparticles driven by selective wettability of silver nanostructures. Nat. Commun. 2014, 5, 2994. [CrossRef]

39. Zhan, P.; Wen, T.; Wang, Z.-g.; He, Y.; Shi, J.; Wang, T.; Liu, X.; Lu, G.; Ding, B. DNA Origami Directed Assembly of Gold Bowtie Nanoantennas for Single-Molecule Surface-Enhanced Raman Scattering. Angew. Chem. Int. Ed. 2018, 57, 2846-2850. [CrossRef]

40. Li, T.; Wu, X.; Liu, F.; Li, N. Analytical methods based on the light-scattering of plasmonic nanoparticles at the single particle level with dark-field microscopy imaging. Analyst 2017, 142, 248-256. [CrossRef] 
41. Haes, A.J.; Duyne, R.P.V. Preliminary studies and potential applications of localized surface plasmon resonance spectroscopy in medical diagnostics. Expert Rev. Mol. Diagn. 2004, 4, 527-537. [CrossRef] [PubMed]

42. Sato, H.; Kawasaki, M.; Kasatani, K.; Katsumata, M.-a. Raman Spectra of Some Indo-, Thia- and Selena-carbocyanine Dyes. J. Raman Spectrosc. 1988, 19, 129-132. [CrossRef]

43. Novara, C.; Chiadò, A.; Paccotti, N.; Catuogno, S.; Esposito, C.L.; Condorelli, G.; De Franciscis, V.; Geobaldo, F.; Rivolo, P.; Giorgis, F. SERS-active metal-dielectric nanostructures integrated in microfluidic devices for label-free quantitative detection of miRNA. Faraday Discuss. 2017, 205, 271. [CrossRef] [PubMed]

44. Kim, W.; Lee, S.H.; Kim, S.H.; Lee, J.-C.; Moon, S.W.; Yu, J.S.; Choi, S. Highly Reproducible Au-Decorated ZnO Nanorod Array on a Graphite Sensor for Classification of Human Aqueous Humors. ACS Appl. Mater. Interfaces 2017, 9, 5891-5899. [CrossRef]

45. Gebhardt, C.; Lehmann, M.; Reif, M.M.; Zacharias, M.; Gemmecker, G.; Cordes, T. Molecular and Spectroscopic Characterization of Green and Red Cyanine Fluorophores from the Alexa Fluor and AF Series. ChemPhysChem 2021, 22, 1566-1583. [CrossRef] [PubMed] 